\title{
Análises e correlações entre resultados histopatológicos, morbidade e mortalidade registrados nos laudos necroscópicos do SVOI de Ribeirão Preto - SP, 2014-2016
}

Analysis and correlations between histopathological results, morbidity and mortality recorded in the necroscopic reports of the SVOI of Ribeirão Preto - SP, 2014-2016

Análisis y correlaciones entre resultados histopatológicos, morbilidad y mortalidad registradas en los informes necroscópicos del SVOI de Ribeirão Preto - SP, 2014-2016

\author{
Isabella Sousa Rocha \\ ORCID: https://orcid.org/0000-0001-6894-9990 \\ Instituto Tocantinense Presidente Antônio Carlos, Brasil \\ E-mail: isabella96srocha@gmail.com \\ Maria Eduarda Nunes da Silva \\ ORCID: https://orcid.org/0000-0003-3996-2584 \\ Instituto Tocantinense Presidente Antônio Carlos, Brasil \\ E-mail: eduarda.nsmed@gmail.com \\ Mariana do Prado Borges \\ ORCID: https://orcid.org/0000-0002-7032-8920 \\ Instituto Tocantinense Presidente Antônio Carlos, Brasil \\ E-mail: marianapb_96@hotmail.com \\ Arthur Alves Borges de Carvalho \\ ORCID: https://orcid.org/0000-0003-0715-5403 \\ Instituto Tocantinense Presidente Antônio Carlos, Brasil \\ E-mail: arthursvo@gmail.com \\ Edson Garcia Soares \\ ORCID: https://orcid.org/0000-0001-7295-3415 \\ Universidade de São Paulo, Brasil \\ E-mail: egsoares@fmrp.usp.br
}

\begin{abstract}
Resumo
Objetivo: Analisar os registros de amostras biológicas coletadas para exame histopatológico nos laudos necroscópicos do Serviço de Verificação de Óbitos de Ribeirão Preto (SVOI-RP) e correlacionar registros de resultados histopatológicos com os registros de causas de mortalidade e de morbidade. Metodologia: Estudo analítico observacional transversal em dados coletados nos laudos necroscópicos, concluídos pelos médicos patologistas e arquivados do SVOI-SP, entre 2014 e 2016. Resultados: Foram estudados 702 laudos necroscópicos, com 1652 registros de causas de mortalidade, 625 causas de morbidade e de 291 amostras coletadas para histopatologia. Discussão: Foram utilizados 28 registros de amostras coletadas para histopatologia com autólise, não havendo correlação significativa com quantidade e qualidade das causas de morbidade e mortalidade. Houve um maior registro de causa de mortalidade naqueles laudos sem registros de amostras coletadas. Laudos com registros superiores a sete amostras apresentaram menor quantidade de morbidades. Conclusão: Não houve necessidade de análise histopatológica para a definição de diagnósticos de mortalidade e morbidade. Padronizar a coleta de amostras poderá dirimir custos, mesmo sem o impacto da autólise na quantidade e qualidade das causas de mortalidade e morbidade no SVOI de Ribeirão Preto, São Paulo.
\end{abstract}

Palavras-chave: Bancos de espécimes biológicos; Autólise; Autópsia; Patologia.

\begin{abstract}
Objective: To analyze the records of biological samples collected for histopathological examination in the necroscopic reports of the Service of Ribeirão Preto (SVOI-RP) and to correlate records of histopathological results with records of causes of Death Verification mortality and morbidity. Methodology: Cross-sectional observational analytical study on data collected in necropsy reports, completed by pathologists and filed at the SVOI-SP, between 2014 and 2016. Results: 702 necroscopic reports were studied, with 1652 records of causes of mortality, 625 causes of morbidity and of 291 samples collected for histopathology. Discussion: Twenty-eight records of samples collected for histopathology with autolysis were used, with no significant correlation with quantity and quality of causes of morbidity and mortality. There was a greater record of cause of mortality in those reports without records of collected samples. Reports with records of more than seven samples had a lower amount of morbidities. Conclusion: There was no need for histopathological analysis to define diagnoses of mortality and morbidity. Standardizing the collection of
\end{abstract}


samples could reduce costs, even without the impact of autolysis on the quantity and quality of causes of mortality and morbidity in the SVOI in Ribeirão Preto, São Paulo.

Keywords: Biological specimen banks; Autolysis; Autopsy; Pathology.

\section{Resumen}

Objetivo: Analizar los registros de muestras biológicas recolectadas para examen histopatológico en los informes necroscópicos del Servicio de Verificación de Defunciones de Ribeirão Preto (SVOI-RP) y correlacionar registros de resultados histopatológicos con registros de causas de mortalidad y morbilidad. Metodología: Estudio analítico observacional transversal sobre datos recolectados en informes de necropsias, completado por patólogos y archivados en el SVOI-SP, entre 2014 y 2016. Resultados: Se estudiaron 702 informes necroscópicos, con 1652 registros de causas de mortalidad, 625 causas de morbilidad y de 291 muestras recogidas para histopatología. Discusión: Se utilizaron 28 registros de muestras recolectadas para histopatología con autólisis, sin correlación significativa con la cantidad y calidad de las causas de morbilidad y mortalidad. Hubo mayor registro de causa de mortalidad en aquellos informes sin registros de muestras recolectadas. Los informes con registros de más de siete muestras tuvieron una menor cantidad de morbilidades. Conclusión: No hubo necesidad de análisis histopatológico para definir diagnósticos de mortalidad y morbilidad. La estandarización de la recolección de muestras podría reducir los costos, incluso sin el impacto de la autólisis en la cantidad y calidad de las causas de mortalidad y morbilidad en el SVOI en Ribeirão Preto, São Paulo.

Palabras clave: Bancos de muestras biológicas; Autólisis; Autopsia; Patología.

\section{Introdução}

Os indicadores de mortalidade são cruciais na avaliação do perfil epidemiológico das causas de óbitos, da situação de saúde das populações e dos serviços de saúde, além de promoverem planejamento e implementação de políticas de saúde. Contudo, a elevada taxa de óbitos por causas mal definidas (CMD) pode comprometer a análise de dados sobre mortalidade e morbidade, comprometendo a fidelidade estatística (Cunha, Teixeira \& França, 2010).

No Brasil, dados sobre óbitos que alimentam o Sistema de Informações sobre Mortalidade (SIM) provêm de informações atestadas nas Declarações de Óbito (DO), preenchida por ato médico. Para investigação necroscópica e elucidação de óbitos com causa natural mal definida, aqueles que seriam atestados por médicos substitutos ou assistente com informações sobre sinais e sintomas ou "garbage codes", podem ser realizadas necropsias nos Serviço de Verificação de Óbitos (SVO) (Rozman, 2007).

Os 43 SVOs habilitados que compõem a Rede Nacional de Serviços de Verificação de Óbito e Esclarecimento da Causa Mortis, instituída pelo Governo Federal em 2006, objetivam elucidar as causas de óbitos naturais mal definidas, com ênfase nas causas de interesse epidemiológico. Alguns serviços oferecem remoção, tanatologia, tanatopraxia, acolhimento social e psicológico aos familiares enlutados. (Brasil, 2006).

O município de Ribeirão Preto - SP possui o SVOI-RP e o SVOR-RP. O Serviço de Verificação de Óbitos do Interior (SVOI-RP), é uma unidade da USP localizada no Centro de Medicina Legal, coordenada pelo departamento de Patologia e Medicina Legal da Faculdade de Medicina de Ribeirão Preto (FMRP/USP) que fornece materiais didáticos para os alunos de medicina da USP e realiza necropsias apenas de óbitos ocorridos por causas naturais no município de Ribeirão Preto. O Serviço de Verificação de Óbitos Regional (SVOR-RP), localizado no Hospital das Clínicas da FMRP, está sob a responsabilidade do Serviço de Patologia do HCFMRP/USP, que realiza biópsias, citologias e necropsias de municípios pertencentes à Divisão Regional da FMRP/USP Saúde de Ribeirão Preto (FMRP/USP, 2019).

Os exames necroscópicos têm papel fundamental na melhoria da qualidade das informações a respeito das causas de óbitos, por fornecer mais elementos para seu esclarecimento. No entanto, a confiabilidade do diagnóstico pode ser comprometida pela qualidade das amostras coletadas que, por sua vez, tem relação com a ausência de padronização dos procedimentos operacionais da necropsia nos SVO (Rozman, 2007).

Para elaborar as causas de mortalidade e morbidade nos laudos necroscópicos do SVOI, os patologistas podem 
realizar análise histopatológica das amostras biológicas coletadas durante a necropsia. Mesmo após a coleta da amostra, o patologista pode definir diagnósticos de mortalidade e morbidades pela anatomopatologia, e não realizar histopatologia. Quando o patologista envia amostra para análise histopatológica, alguns resultados são registrados como autólise. A autólise é um fenômeno de decomposição tecidual que pode estar associada à inadequados procedimentos de coleta, acondicionamento e transporte das amostras biológicas oriundas de necropsia.

Falhas ou ausência de procedimentos necroscópicos padronizados que envolvem coleta, acondicionamento e transporte de amostras biológicas podem resultar na presença de autólise na histopatologia, impactando a quantidade e qualidade dos resultados histopatológicos e dos diagnósticos de mortalidade e morbidade nos laudos necroscópicos do SVOIRP.

A padronização de procedimentos operacionais pode dirimir custos e trazer maior acreditação ao serviço. Relacionar quantidade e qualidade dos registros histopatológicos com autólise e com quantidade de registros de causas de mortalidade e de morbidade nos laudos necroscópicos pode motivar padronização de procedimentos operacionais dentro do SVOI-RP e dos demais serviços da Rede Nacional.

\section{Metodologia}

Trata - se de um estudo analítico observacional transversal executado nos registros digitais de laudos necroscópicos finalizados pelos médicos patologistas entre 2014 e 2016 e arquivados no Serviço de Verificação de Óbitos do Interior de Ribeirão Preto. O estudo correlacionou a qualidade e quantidade de amostras biológicas coletadas e registradas nos laudos necroscópicos com a quantidade de causas de mortalidade e de morbidade registradas.

Os dados foram coletados a partir dos registros em laudos necroscópicos digitalizados concluídos pelos médicos patologistas e arquivados. A população do estudo consiste nos óbitos ocorridos em Ribeirão Preto e submetidos à necropsia no SVOI-RP, entre 2014 e 2016. Foram analisados 234 registros de laudos digitais por ano de elaboração do laudo, totalizando 702 registros de laudos digitais necroscópicos analisados, para o período 2014 a 2016.

Os números foram obtidos de acordo com o cálculo para obtenção do tamanho de amostra: $N=\frac{n 0}{1+\frac{n(n 0-1)}{N}}$, onde "N" corresponde ao valor da população total (ou seja 600 laudos, pois é a média de laudos necroscópicos produzidos por ano no SVOI-RP) e que "n0" é dado pelo cálculo: $n 0=\frac{z h^{2}}{4 d^{2}}$, sendo que o numerador corresponde ao grau de confiança e o "d" a margem de erro da pesquisa.

Assim, com uma confiabilidade de $95 \%$ e margem de erro de 5\%, obtivemos o seguinte cálculo:

$$
\begin{gathered}
n 0=\frac{z k^{2}}{4 d^{2}}=\frac{1,96^{2}}{4(0,05)^{2}}=\frac{3,8416}{0,01}=384,16 \\
N=\frac{n 0}{1+\frac{n 0-1}{N}}=\frac{384,16}{1+\frac{384,16-1}{600}}=234,5
\end{gathered}
$$

$\mathrm{N}=234$ laudos por ano, totalizando 702 laudos entre 2014 e 2016.

Neste estudo foram consideradas as variáveis: Quantidade amostras biológicas coletadas por necropsia, qualidade das amostras biológicas, quantidade de diagnósticos de causas de mortalidade e quantidade de diagnósticos de causas de morbidade. 
Os dados coletados foram organizados em planilha do Excel® 2013, sendo dispostas nas colunas as variáveis estudadas e nas linhas a identificação de cada amostra (n) conforme iniciais do nome, idade e sexo.

Para a verificação da Normalidade, foi utilizado o software SAS (SAS, 2013) por meio dos testes de KolmogorovSmirnov (K-S) e de Shapiro-Wilk (S-W) a 5\% de significância. Estes testes forneceram o parâmetro valor de prova (valor-p, pvalue ou significância), que pode ser interpretado como a medida do grau de concordância entre os dados e a hipótese nula (H0), sendo H0 correspondente à distribuição Normal. Quanto menor for o valor de p, menor é a consistência para aceitar a hipótese nula. Depois da determinação da normalidade, foi implementado uma Análise de Variância (ANOVA - oneway), por meio do procedimento npar1way, utilizando o teste dos ranks de Wilcoxon e de Kruskal-Wallis, os quais permitiram testar o efeito da quantidade de amostras biológicas coletadas e registradas nos laudos necroscópicos do SVOI de Ribeirão Preto, com a quantidade de registros sobre as causas de mortalidade e de morbidade. Assim, cada observação Yij, que representa os valores de HAS, pode ser decomposta conforme o modelo abaixo:

$$
\mathrm{Yij}=\mu+\mathrm{Vi}+\varepsilon \mathrm{ij}
$$

Em que:

Yij é a observação do i-ésimo tratamento na j-ésima unidade experimental; $\mu$ é o efeito constante (média geral);

Vi é o efeito do i-ésima variável;

عij é o efeito do erro aleatório associado a i-ésima variável i com o erro j.

Para avaliação da qualidade das amostras biológicas foi utilizado o critério presença de autólise registrada nos resultados histopatológicos dos laudos necroscópicos digitalizados.

Adicionalmente, foi aplicada a análise de correlação para identificar o grau de relacionamento linear entre as variáveis de interesse, no caso do presente estudo, os registros de número de morbidade e mortalidade com a quantidade de amostra e o número de autólise identificada. O modelo que adotado nesta análise pode ser representado como:

$$
r=\frac{\sum X i Y i x \frac{\left(\sum X i Y i\right)}{n}}{\sqrt{\sum X i^{2}-\frac{\left(\sum X i\right)^{2}}{n} x \sqrt{\sum Y i^{2}-\frac{\left(\sum Y i\right)^{2}}{n}}}}
$$

Em que:

$\mathrm{r}=$ representa o coeficiente de correlação entre as variáveis utilizadas na análise;

$\sum \mathrm{XiYi}=$ somatório entre o produto das duas variáveis utilizadas na análise;

$\mathrm{n}=$ número de observações do banco de dados;

$\sum \mathrm{Xi}^{2}=$ somatório dos valores da variável $\mathrm{X}$ elevado ao quadrado;

$\sum \mathrm{Yi}^{2}=$ somatório dos valores da variável Y elevado ao quadrado;

Os resultados gerados nas análises foram organizados em tabelas e gráficos para melhor visualização.

\section{Resultados}

Foram estudados 702 registros digitais de laudos necroscópicos, com 1652 causas de mortalidade (N1) registradas com média de $2.35 \pm 1.02$ (C.V. 43,27\%). O valor mínimo de N1 foi 1, valor máximo: 4, $1^{\circ}$ Quartil (Q1): 1, Mediana: 2, $3^{\circ}$ quartil (Q3): 3, moda: 3, assimetria: 0.09, curtose: -1.13, definindo-se uma curva de distribuição leptocúrtica. A maioria dos laudos apresentou 3 registros de diagnósticos de mortalidade.

O número de registros de causas de morbidades (N2) foi 625 com média de $0.89 \pm 1.02$ (C.V. 114,38\%). O valor mínimo foi 0, valor máximo: 6, $1^{\circ}$ Quartil (Q1): 0, Mediana: 1, 3 quartil (Q3): 2, moda: 0, assimetria 1.22, curtose: 1.70, definindo-se uma curva de distribuição platicúrtica. A maioria dos laudos não apresentou registros de causas de morbidade. 
O número de registros de amostras coletadas para histopatologia (A1) foi 291 com média de $0.41 \pm 1.64$ (C.V. $400 \%)$. O valor mínimo foi 0, valor máximo: $10,1^{\circ}$ Quartil (Q1): 0, Mediana: 0, $3^{\circ}$ quartil (Q3): 0, moda: 0, assimetria: 3.97, curtose: 14.72, definindo-se uma curva de distribuição platicúrtica e 33 laudos, que apresentaram pelo menos um registro de coleta de amostras para histopatologia, não tinham registros de autólise.

Dos 702 registros digitais de laudos necroscópicos, 45 possuíam pelo menos um registro de coleta de amostras biológicas para histopatologia. Foi encontrado o total de 28 registros de autólises (A2) dentre 12 dos 45 , com média: $0.62 \pm$ 1.34 (C.V. 216.13\%). O valor mínimo foi 0, valor máximo: 7, $1^{\circ}$ Quartil (Q1): 0, Mediana: 0, $3^{\circ}$ quartil (Q3): 1, moda: 0, assimetria: 3.01, curtose: 11.24, definindo-se uma curva de distribuição platicúrtica.

Figura 1: Quantidade de registros segundo a quantidade de amostras histopatológica, quantidade de autólises, registros de mortalidade e registro de morbidade.


Fonte: Autores.

Após a aplicação dos testes de normalidade, pode-se observar que as variáveis, foco do presente estudo, não apresentaram distribuição normal (Quadro 1), evidenciado pelo valor-p $\leq \alpha$ (rejeita-se H0), ou seja, não se pode admitir que o conjunto de dados em questão tenha distribuição Normal. A condição de normalidade é um dos pré-requisitos para teste paramétricos. 
Quadro 1: Teste de normalidade de Kolmogorov-Smirnov e Shapiro-Wilk para as variáveis A1_histopatologia, A2_autolise, N1_mortalidade e N2_morbidade.

\begin{tabular}{|c|c|c|c|c|}
\hline \multirow{2}{*}{ Variáveis } & \multicolumn{4}{|c|}{ Tests of Normality } \\
\cline { 2 - 5 } & Kolmogorov-Smirnov & \multicolumn{2}{|c|}{ Shapiro-Wilk } \\
\cline { 2 - 5 } & Statistic & PValue & Statistic & pValue \\
\hline A1_histopatologia & 0,53 & 0,000 & 0,27 & 0,000 \\
\hline A2_autolise & 0,41 & 0,000 & 0,54 & 0,000 \\
\hline N1_mortalidade & 0,20 & 0,000 & 0,87 & 0,000 \\
\hline N2_morbidade & 0,26 & 0,000 & 0,80 & 0,000 \\
\hline
\end{tabular}

Fonte: Autores.

Quanto ao teste de correlação, apenas duas variáveis foram significativas, no entanto, com coeficiente muito baixo. Houve correlação negativa muito fraca entre a quantidade amostras histopatológicas e causas de mortalidade, significância $\mathrm{p}<0.001$, demonstrando que o aumento do número de registros para amostras biológicas coletadas relaciona-se com a diminuição do número de registros para diagnósticos de mortalidade.

Entre as causas de mortalidade e causas de morbidade evidenciaram também uma correlação positiva muito fraca, significância $\mathrm{p}<0.001$, indicando que o aumento no número de causas de morte relaciona-se com aumento de causas de morbidade. (Quadro 2).

Quadro 2: Teste de Correlação de Spearman’s para as variáveis N1 (causas de mortalidade) N2 (causas de morbidade), A1_histopatologia (número de amostras teciduais) A2_autolise (número de autólises).

\begin{tabular}{|c|c|c|c|c|c|}
\hline & & A1_histopatologia & A2_autolise & N1_mortalidade & N2_morbidade \\
\hline \multirow{2}{*}{ A1_histopatologia } & Correlation Coefficient & 1,00 & 0,07 & $-0,11$ & $-0,06$ \\
\cline { 2 - 6 } & pValue & & 0,66 & 0,00 & 0,12 \\
\hline \multirow{2}{*}{ A2_autolise } & Correlation Coefficient & 0,07 & 1,00 & $-0,11$ & $-0,23$ \\
\cline { 2 - 6 } & pValue & 0,66 & & 0,47 & 0,12 \\
\hline \multirow{2}{*}{ N1_mortalidade } & Correlation Coefficient & $-0,11$ & $-0,11$ & 1,00 & 0,21 \\
\cline { 2 - 6 } & pValue & 0,00 & 0,47 & & 0,00 \\
\hline \multirow{2}{*}{ N2_morbidade } & Correlation Coefficient & $-0,06$ & $-0,23$ & 0,21 & 1,00 \\
\cline { 2 - 6 } & pValue & 0,12 & 0,12 & 0,00 & \\
\hline \multicolumn{7}{|c|}{ Correlation is significant at the 0.01 level (2-tailed). } \\
\hline
\end{tabular}

Fonte: Autores.

O teste Kruskal-Wallis mostrou que há efeito da quantidade de amostras histopatológicas coletadas sobre o número de causas de mortalidade $(\mathrm{p}=0,006)$ e de morbidade $(\mathrm{p}=0,016)$. Quanto ao efeito da quantidade de autólise sobre o número de causas de mortalidade e de morbidade, os resultados não foram significativos para ambos. (Quadro 3).

Realizando as comparações em pares para verificar onde encontrava-se o efeito da quantidade de amostra histopatológicas, observou-se que existe uma diferença significativa $(\mathrm{p}=0,009)$ entre os pares 0 amostras e $7+$ amostras. Analisando a figura 2 pode-se afirmar que a média de causas de mortalidade descrita foi maior para os laudos que não tinham registros de amostras biológicas coletadas para estudo histopatológico.

Quanto aos efeitos da quantidade de amostra histopatológicas com número de causas de morbidade a diferença foi significativa nos seguintes pares, $7+$ amostras e 0 amostra $(p=0,014)$ e $7+$ amostras com 1 a 6 amostras $(p=0,037)$, com esse resultado pode-se afirmar que quando o número de amostra foi igual ou superior a 7 o número de causas de morbidade diminuiu (Figura 3). 
Quadro 3: Análise de Variância do efeito da quantidade de amostras histopatológicas entre as variáveis utilizadas neste trabalho, gerados a partir do teste de Kruskal-Wallis.

\begin{tabular}{|c|c|c|c|c|c|}
\hline & Resultado & A1_histopatologia & A2_autolise & N1_mortalidade & N2_morbidade \\
\hline \multirow{2}{*}{ Histopatologia } & Chi-Square & - & 1,52 & 10,2 & 8,33 \\
\cline { 2 - 6 } & pValue & - & 0,217 & 0,006 & 0,016 \\
\hline \multirow{2}{*}{ Autólise } & Chi-Square & 174,85 & - & 8,086 & 6,616 \\
\cline { 2 - 6 } & pValor & 0,00 & - & 0,088 & 0,158 \\
\hline
\end{tabular}

Fonte: Autores.

Figura 2: Boxplot resultado teste Kruskal-Wallis para variável mortalidade.

Independent-Samples Kruskal-Wallis Test

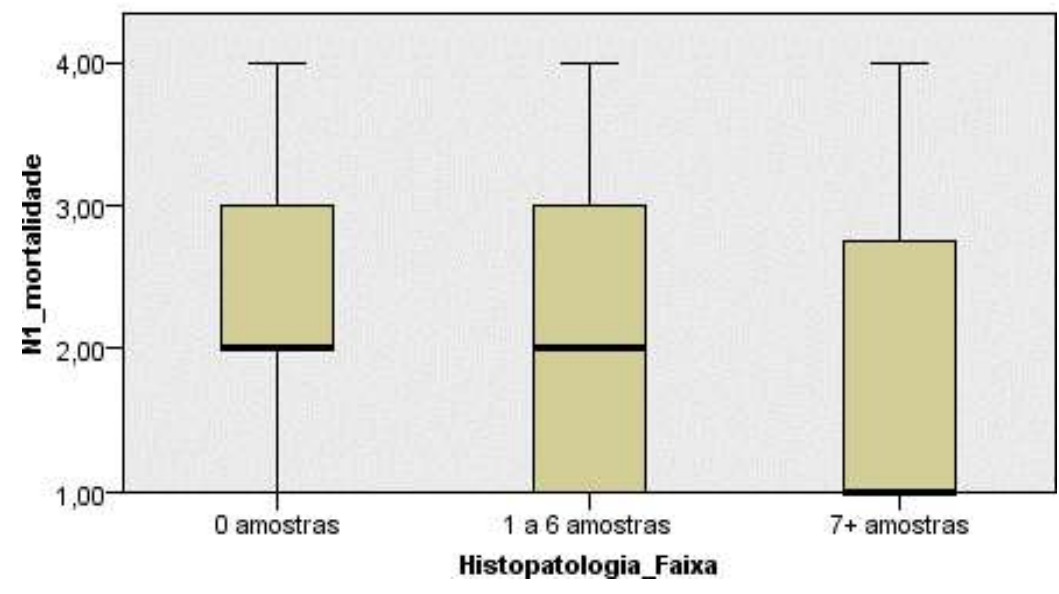

Fonte: Autores.
Figura 3: Boxplot

Wallis para

\section{Independent-Samples Kruskal-Wallis Test}



resultado teste Kruskalvariável morbidade.

Fonte: Autores.

\section{Discussão}

Segundo a Organização Mundial da Saúde (OMS), a presença de dados precisos, oportunos e de qualidade são essenciais para fidelidade das informações em saúde, programar treinamentos específicos de recursos humanos e direcionamento de recursos e implementação de políticas públicas de saúde. Informações incompletas sobre morbimortalidade 
podem afetar credibilidade e confiabilidade dos sistemas que armazenam, processam e analisam estatísticas vitais (WHO, 2015; Santos Filho et al., 2017).

O SVO, pertencente à Rede Nacional, é regido pela Portaria $\mathrm{n}^{\circ} 1.405$ de 29 de julho de 2006, integrando assim o Sistema Nacional de Vigilância em Saúde do Ministério da Saúde. Por meio de necropsias, os SVO elucidam casos de óbitos naturais mal definidos, diferente do Instituto Médico legal (IML) que atua como órgão de Segurança Pública determinando causas e circunstâncias de óbitos por causas externas. (Brasil, 2006).

Os números de óbitos examinados e atestados pelos SVO variam entre alguns serviços. No Distrito Federal foram atestados 1720 casos pelo SVO-DF, somente em 2019. Carvalho, 2016 refere que no triênio 2010, 2011 e 2012 foram examinados 818 óbitos no SVO do Estado do Tocantins, enquanto no SVOI-RP foram examinados e atestados 702 óbitos de 2014 à 2016. Os diferentes números de óbitos examinados e atestados pelos SVO podem estar relacionados a diferentes números de habitantes, diferentes áreas de abrangências municipais ou estaduais, qualidade dos fluxos de remoções cadavéricas públicas ou terceirizadas, sensibilização da classe médica e de familiares enlutados sobre vigilância dos óbitos mal definidos.

Comorbidade é a presença de duas afecções ou moléstias, ao mesmo tempo, no paciente. Multimorbidade consiste em presença de mais de duas doenças ao mesmo tempo. A presença de comorbidades causa dificuldades diagnósticas e está associada a falências terapêuticas. Identificar e analisar comorbidades registradas nos laudos necroscópicos do SVO permite relacionar, eventuais dificuldades médicas diagnósticas e terapêuticas, com causas de mortalidade e circunstâncias que envolvem óbitos naturais mal definidos (Sanches, Assunção \& Hetem, 2005; Tugwell \& Knottnerus, 2019).

Laudos necroscópicos com ausência de registros de comorbidades podem estar associados, dentre outros fatores, a ausência de acolhimento do familiar enlutado no SVOI. A presença de acolhimento ao familiar enlutado promoveria coleta de informações sobre as condições de saúde, sociais e econômicas do falecido, favorecendo a identificação de comorbidades. No Serviço de Verificação de Óbitos do Estado do Tocantins (SVO-TO), o setor de acolhimento familiar enlutado suporta estudos sobre perfil epidemiológico dos óbitos examinados (Martins \& Carvalho, 2020).

A necropsia consiste em procedimento de análise anatomopatológica com mensuração de dimensões, coleta de material biológico e processamentos (desidratação, clarificação, inclusão e cortes micrométricos) para estudos histopatológicos (Caputo, Gitirana \& Manso, 2010). Apesar de métodos diagnósticos padrão ouro para diversas doenças, o sub- registro ou subrealização da histopatologia, pode significar uma baixa acurácia diagnóstica final (Rio De Janeiro, 2020). O método mais preciso para elucidar a causa da morte é a necropsia com analise histopatológica (Roulson, Benbow, \& Hasleton, 2005).

No SVOI, 657 laudos $(93,59 \%)$ não apresentaram registros de coleta de amostras para exame histopatológico. O resultado encontrado pode estar associado a expertise dos patologistas em elucidar causas de óbitos naturais mal definidas por meio da anatomopatologia exclusiva, entretanto o método mais preciso para elucidar a causa da morte é a necropsia com analise histopatológica (Roulson, Benbow, \& Hasleton, 2005).

A técnica necroscópica no SVOI com metodologia de coletar, acondicionar em formol e proceder com a histopatologia somente quando o patologista julgar pertinente, pode contribuir para o baixo número de registros de resultados de amostras coletadas para histopatologia. Análise anatomopatológica exclusiva pode negligenciar doenças infecciosas, embolias e isquemias, fortalecendo a recomendação para análise histopatológica de órgãos alvo como coração, pulmão, fígado e rins (Rous, 2019).

Nos laudos estudados em Ribeirão Preto foram encontrados 291 registros de amostras coletadas para exame histopatológico, com média de 0,41 $\pm 1,64$, por necropsia, porém o manual sobre "Autópsias da Sociedade Brasileira de Patologia" preconizar que sejam confeccionadas cerca de 40 lâminas para que ocorra uma representação ideal de todos os órgãos (Echenique et al., 2002). No SVO-TO, quando coletadas mais de 7 amostras biológicas para exame histopatológico por 
necropsia, não há impacto na quantidade de causas de mortalidade registradas nos laudos necroscópicos (Carvalho et al, 2020).

A autólise é a autodigestão enzimática de células, característica do "post mortem" (Farlex Partner Medical Dictionary, 2012). Causas de autólise relacionam-se com obesidade do falecido, calor, necropsias com exposição excessiva do cadáver à luz brilhante, quantidade de sangue não lavado, acumulado nos órgãos ou tecidos e demora em fixar o órgão ou tecido após retirada do cadáver (Brenda \& Waters, 2009).

Tecidos preservados em ambientes frios apresentam menores efeitos da autólise (Cocariu et al, 2016). O baixo número de registros de autólises pode ser atribuído ao pequeno número de registros de coleta de amostras biológicas sustentando o insignificante efeito do número de registros de autólises sobre o número de registros de causas de morbidade e mortalidade.

Diversas técnicas de coleta de amostras para diferentes tipos de diagnósticos preservam o tecido coletado evitando artefatos e intepretações errôneas (Pires, Travassos, Gama \& Carreira, 2012). Apesar do percentual de 26,6\% registros de autólise nos laudos do SVOI-RP, não foi avaliado o impacto da qualidade da coleta, acondicionamento e transporte de amostras biológicas sobre o número reduzido de registros de autólises nos laudos.

Hasleton et al, 2005 refere que pelo menos metade dos exames necroscópicos produzem resultados discrepantes das hipóteses diagnósticas clinicas de morte. Mais de $20 \%$ dos achados de necropsia clinicamente inesperados, e 5\% dos achados principais podem ser diagnosticados corretamente apenas por exames histopatológicos. No SVOI-RP, a maior média de registros de causas de mortalidade foi encontrada nos laudos que não tinham registros de coleta de amostras biológicas para exames histopatológicos (Roulson, Benbow, \& Hasleton, 2005).

Mesmo com alto nível médico pericial dentro do SVO-RP, causas de mortalidade e de mortalidade deixam de ser diagnosticadas quando não se correlaciona a história clínica, a anatomia patológica e a histopatologia. Dos 702 laudos estudados, $45(6,41 \%)$ apresentaram pelo menos um registro de coleta de amostras biológicas para a histopatologia. Estabelecer procedimentos operacionais padrão de coleta de amostras biológicas poderia padronizar coletas de amostras dos exames necroscópicos e impactar na quantidade de causas de mortalidade e morbidade.

\section{Conclusão}

A análise dos laudos necroscópicos do SVOI em Ribeirão Preto revelou que o número de amostras com autólise registradas foi de $26,66 \%$. Não houve correlação significativa entre a quantidade de registro de autólise e o número de diagnósticos de morbidade e mortalidade. $\mathrm{O}$ aumento da quantidade de amostras histopatológicas coletadas por laudo impactou na redução do número de registros de diagnósticos de mortalidade. Quando a quantidade de amostras histopatológicas foi superior a sete amostras, evidenciou-se diminuição no número de causas de morbidade. Dos laudos analisados 657 (93,59\%) não apresentou registros de amostras histopatológicas coletadas. Os laudos sem amostras coletadas apresentaram maior média de causas de mortalidade que os laudos com registros de coletas de amostras. Pode se estabelecer um padrão quantitativo de amostra coletadas para análises histopatológicas, pode-se estabelecer no SVOI em Ribeirão Preto.

\section{Referências}

Autolysis. (n.d.) Farlex Partner Medical Dictionary. (2012). https://medical-dictionary.thefreedictionary.com/autolysis

Brasil. (2006). Portaria n 1.405 de 29 de junho de 2006. Institui a Rede Nacional de Serviços de Verificação de Óbito e Esclarecimento da Causa Mortis (SVO). Diário Oficial da República Federativa do Brasil, Brasília.

Brenda, L. \& Waters, M. D. (2009). Handbook of Autopsy Practice (4a ed.). Humana Press.

Caputo, L. F. G., Gitirana, L. B. \& Manso, P. P. A. (2010). Técnicas Histológicas. In: Conceitos e Métodos para a formação de Profissionais em Laboratórios de Saúde. Escola Politécnica de Saúde Joaquim Venâncio, p.89-188. 
Carvalho, A. B. A., Soares, E. G., Costa, L. R., Silva, P. D. L., Gama, M. V., Bessa, N. G. F., Almeida, J., \& Miranda, W. D. (2020). Análise de Amostras Biológicas em Laudos Necroscópicos do Serviço de Verificação de Óbitos do Estado do Tocantins, Brasil. Journal of Advances in Medicine and Medical Research, 31(11):1-13. https://doi.org/10.9734/jammr/2019/v31i1130331

Cocariu, E., Mageriu, V., Stăniceanu, F., Bastian, A., Socoliuc, C. \& Zurac, S.(2016).Correlations Between the Autolytic Changes and Postmortem Interval in Refrigerated Cadavers. Romanian Journal of Internal Medicine,54(2) 105-112. https://doi.org/10.1515/rjim-2016-0012

Cunha, C. C., Teixeira, R. \& França, E. (2017). Avaliação da investigação de óbitos por causas mal definidas no Brasil em 2010. Epidemiologia e Serviços de Saúde, 26:19-30.

Echenique, L.S. et al. (2002). Correlação entre achados macro e microscópicos em 200 autópsias consecutivas: análise do valor custo/benefício do estudo histopatológico completo das autópsias. J. Bras. Patol. Med. Lab., 38(3):219-224.

FAMEMA. Faculdade de Medicina de Marília. Secretaria de Desenvolvimento Econômico, Ciência, Tecnologia e Inovação. Solicitação de Necropsia. Marília, [s.d.].

Ferreira, E. B., Rocha, M. C. \& Mequelino, D. B. (2012). Monte Carlo evaluation of the ANOVA's F and Kruskal-Wallis tests under binomial distribution. Sigmae, 1(1):126-139.

Jorge, M. H. P. M., Gotlieb, S. L. D. \& Laurenti, R. (2002). O sistema de informações sobre mortalidade: problemas e propostas para o seu enfrentamento I Mortes por causas naturais. Rev. bras. epidemiol., 5(2):197-211.

Laurenti, R. \& Jorge, M. H. P. M. (2015). Atestado de óbito: aspectos médicos, estatísticos, éticos e jurídicos. / Coordenação de. São Paulo: Conselho Regional de Medicina do Estado de São Paulo.

Lebrao, M. L. (1995). Estudos de morbidade: usos e limites. Saúde e Sociedade, 4(1-2):51-57.

Martins, J. F. F. \& Carvalho, A. B. A. (2020). Perfil social e econômico dos necropsiados e respectivas famílias atendidas pelo serviço de verificação de óbito de Palmas- TO. In Costa, N.M., Jonas, L.O. \& Sinimbu Filho, J.M. (Org.). Ciência e Saúde: da Teoria à Prática. Maringá, PR: Uniedusul.

Mendo, C. T. M. (2019). Análise das transferências dos casos de exame necroscópico entre o instituto Médico-Legal (IML) e o serviço de Verificação de Óbito do Interior (SVOI) da Faculdade de medicina de Ribeirão Preto no período de 2009 a 2017. 70f. Tese (doutorado em Ciências) - Faculdade de medicina de Ribeirão Preto, Ribeirão Preto.

Pires, M., Travassos, F. S., Gama, A. \& Carreira, R. P. (2012). BASIC GUIDELINES FOR THE COLLECTION AND SUBMISSION OF NECROPSY SAMPLES.

_OF_NECROPSY_SAMPLES

RIO DE JANEIRO. (2020). Prefeitura municipal. Remessa de peças cirúrgicas para exame histopatológico. Princípios gerais. Portal da Prefeitura do Rio de Janeiro, Rio de Janeiro. http://www0.rio.rj.gov.br/ijv/remessa_pecas.shtm\&gt

Rocha, L.O.S. (2014). Necrópsia e educação médica. Rev. Méd. Minas Gerais: 24(1).

Rous, B. (2019). Guidelines on autopsy practice: Postoperative deaths May 2019.

Roulson, J., Benbow, E. W. \& Hasleton, P. S. (2005). Discrepancies between clinical and autopsy diagnosis and the value of post mortem histology; a metaanalysis and review. Histopathology, 47: 551-559. https://doi.org/10.1111/j.1365-2559.2005.02243.x

Rozman, M.A. (2007). Mortalidade por causa mal definida no Brasil, Estado de São Paulo e Baixada Santista. 1980-2002. Tese de Doutorado. Universidade de São Paulo.

Sanches, R. F., Assunção, S., \& Hetem, L. A. B. (2005). Impacto da comorbidade no diagnóstico e tratamento do transtorno bipolar. Archives of Clinical Psychiatry, 32(1): 71-77. https://doi.org/10.1590/S0101-60832005000700011

Santos Filho, L. A. et al. (2017). Análise da completude dos dados sobre as causas de mortalidade e morbidade no sistema de informação de mortalidade para o estado do Tocantins, 2010-2012. Cereus, 9(1).

Santos, G. E. O. (2019). Cálculo amostral: calculadora on-line. http://www.calculoamostral.vai.la

SVOI FMRP/USP. (2019). O que é um SVO e sua organização no estado de São Paulo. http://svoi.fmrp.usp.br/index.php?option=com_ content $\&$ view $=$ article $\&$ id $=52 \&$ Itemid $=59$

TOCANTINS. (2019). Ministério da Saúde. Manual de Coleta, acondicionamento e transporte de amostras biológicas. Tocantins. https://central3.to.gov.br/arquivo/442521/

Tugwell, P., \& Knottnerus, J. A. (2019). Multimorbidity and Comorbidity are now separate MESH headings. Journal of Clinical Epidemiology, 105:VI-VIII. https://doi.org/10.1016/j.jclinepi.2018.11.019

WHO. (2015). World Health Organization. Human Resources for Health Information System. 